\title{
Atomic and Molecular Gas in the Merger Arp 299
}

\author{
A.G. Polatidis, S. Aalto \\ Onsala Space Observatory, S-43992 Onsala, Sweden
}

\begin{abstract}
We present MERLIN observations of the atomic and molecular gas of IC 694 a member of the merger system Arp 299. The radio continuum of IC 694 is consistent with a disk of synchrotron emission powered by supernovae remnants. The atomic and molecular gas are located in a similarly orientated $250 \mathrm{pc}$ large disk.
\end{abstract}

The merging system Arp 299 (Mrk 171) consists of two interacting galaxies, IC 694 and NGC 3690. Its far-infrared luminosity of $5.2 \times 10^{11} \mathrm{~L}_{\odot}$ places it in the gap between the nearby starburst galaxies (eg M82) and the more distant Ultra Luminous far- InfraRed Galaxies (ULIRGs, eg Arp 220). The system is rich in molecular gas, with bright $\mathrm{CO}$ condensations in the two galactic nuclei, particularly in IC 694 , where the molecular gas mass in the inner $280 \mathrm{pc}$ radius is $\sim 5 \times 10^{9} \mathrm{M}_{\odot}$ and where the $\mathrm{mm}$ molecular line rations reveal a highly disrupted molecular ISM with a bulk temperature $\geq 100 \mathrm{~K}$ (Aalto et al 1997). The detection of $\mathrm{OH}$ megamasers and HI absorption in Arp 299 (Baan \& Haschick 1990, BH90), further emphasises the notion of extreme conditions in the ISM.

In order to investigate the conditions of the atomic and molecular gas in detail we made sub-arcsecond (10-40pc) resolution MERLIN observations of the $\mathrm{HI}$ absorption, the $\mathrm{OH}$ megamaser emission and of the $\lambda 6 \mathrm{~cm}$ Formaldehyde $\left(\mathrm{H}_{2} \mathrm{CO}\right)$ line, and compared them with the $200 \mathrm{pc}$ resolution $\mathrm{CO}$ images of Aalto et al (1997).

The observations were made in the spring of 1998. The observations of the source were phase-referenced to the nearby calibrator $1125+596$. Initial editing and calibration were carried out using standard MERLIN software; further analysis and imaging was performed in AIPS. Here we present preliminary results for the galaxy IC 694 .

\section{The radio continuum, the atomic and molecular gas of IC 694}

The radio continuum shows a $200 \times 250 \mathrm{pc}$ large, diffuse emission, elongated at $\sim 135^{\circ}$, surrounding a brighter, $150 \mathrm{pc}$ long ridge. The overall spectral index is flat between $\lambda 6$ and $21 \mathrm{~cm}$. The structure of IC 694 in not that of a typical corejet seen in bright AGN. Instead, the morphology of IC 694 is consistent with synchrotron emission from electrons accelerated by radio supernovae remnants in a disk, similar to those found in several other ULIRGs (e.g. Mrk 231, Mrk 273 Carilli et al 1998, 2000).

Figure 1 presents the intensity-weighted mean velocity fields and the positionvelocity diagrammes of the $200 \mathrm{pc}$ resolution $\mathrm{CO}$ observations of Aalto et al. (1997) (left) and our HI (middle) and OH (right) observations.

The fairly regular looking spider diagram of the HI absorption reveals a $240 \mathrm{pc}$, rotating disk-like structure with inclination $\sim 60^{\circ}$, elongated along the 

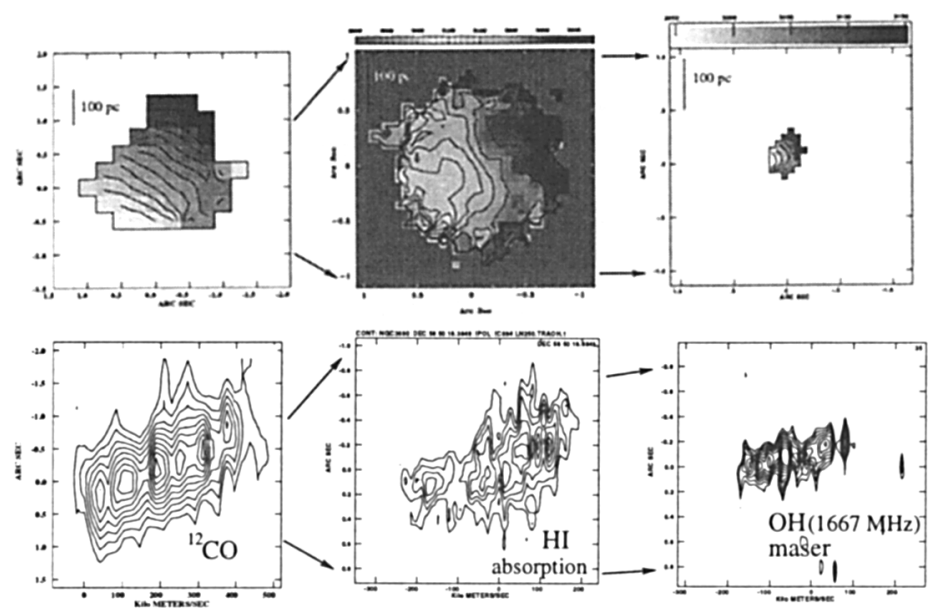

Figure 1. A comparative look of the velocity distribution and the position velocity diagrammes of the $\mathrm{CO}$ emission (left), the $\mathrm{HI}$ absorption (middle) and the $\mathrm{OH}$ megamaser (right) lines of IC 694

major axis of the radio continuum with a velocity gradient of $2094.4 \mathrm{~km} / \mathrm{s} \mathrm{kpc}^{-1}$. The position-velocity diagramme shows several absorption components (with optical depth 0.1-0.5) along the major axis of the continuum, indicating the clumpy nature of the rotating structure. The total $\mathrm{HI}$ column density is $\mathrm{N}_{H I} \sim$ $1.26 \times 10^{20} T_{S}$, implying a mass for the $\mathrm{HI}$ of $\mathrm{M}_{H I}=3.6 \times 10^{6} M_{\odot}$, roughly $1 \%$ of the dynamical mass (for $T_{S}=100 \mathrm{~K}$ ).

$\mathrm{OH}$ megamaser emission is only observed in IC 694 . The $1665 \mathrm{MHz}$ line is almost resolved out, implying that it arises in a more extended region. The 1667 $\mathrm{MHz}$ line emission arises in a $100 \mathrm{pc}$ large rotating structure with a velocity gradient of $2750 \mathrm{~km} / \mathrm{s} \mathrm{kpc}^{-1}$, orientated at $135^{\circ}$. The position-velocity diagramme shows a clumpy nature for the rotating structure similar to that observed in the HI disk.

The atomic and molecular gas in the nucleus of IC 694, is located in flattened rotating structures that have the same axis and similar velocity ranges. The $\mathrm{CO}$ emission and HI absorption seem to occupy the same space $(250 \mathrm{pc})$ suggesting that the HI and molecular gas phases are mixed. It is possible that the HI is a photodissociation product of the molecular gas. The $\mathrm{OH}$ seems to be located further in, in the same rotating disk. The turbulence of the gas is generally high - it often exceeds $100 \mathrm{~km} / \mathrm{s}$. This is consistent with the notion of extremely high gas pressures in the centers of ULIRG galaxies with steep potentials.

\section{References}

Aalto S., Radford S.J.E., Scoville N.Z., Sargent A.I., 1997 ApJ, 475, L107

Baan W. A., Haschick A., 1990 ApJ, 364, 65.

Carilli C.L., Wrobel J.M., Ulvestad J.S. 1998, AJ, 115, 928.

Carilli C.L., Taylor G.B., 2000, ApJ, 532, L95. 